\title{
Visibility of trabecular meshwork by standard and polarization-sensitive optical coherence tomography
}

\author{
Yoshiaki Yasuno \\ Masahiro Yamanari \\ University of Tsukuba \\ Computational Optics Group \\ and \\ Computational Optics and Ophthalmology \\ Group \\ Ibaraki, Japan 305-8573
}

\section{Keisuke Kawana}

University of Tsukuba

Institute of Clinical Medicine

Department of Ophthalmology and

Computational Optics and Ophthalmology

Group

Ibaraki, Japan 305-8573
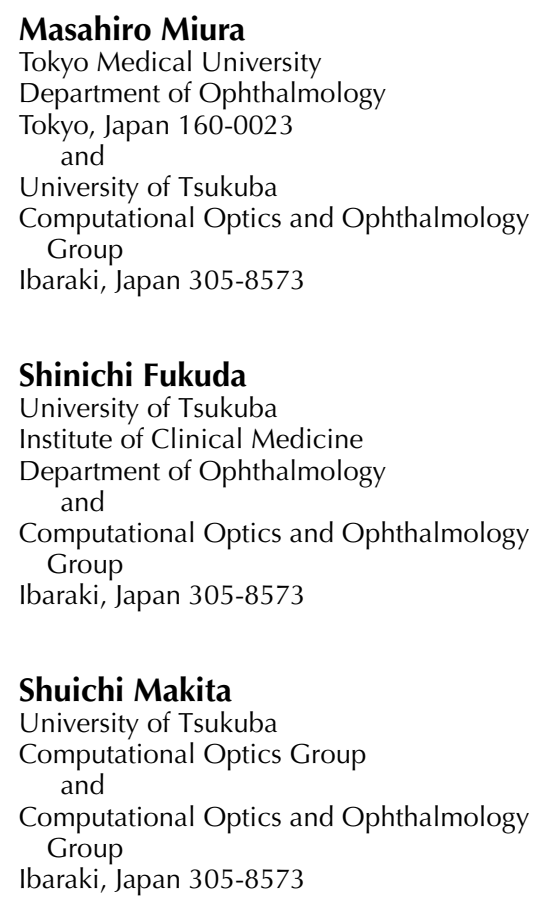

\section{Shingo Sakai}

Kanebo Cosmetics Inc.

Basic Research Laboratory

Kanagawa, Japan 250-0002

\author{
Tetsuro Oshika \\ University of Tsukuba \\ Institute of Clinical Medicine \\ Department of Ophthalmology \\ and \\ Computational Optics and Ophthalmology \\ Group \\ Ibaraki, Japan 305-8573
}

\begin{abstract}
Polarization-sensitive optical coherence tomography (PS-OCT) is known to be advantageous because of its additional tissue-specific contrast of the anterior eye. So far, this advantage has been shown only qualitatively. We evaluate the improved visibility afforded by 3-D PS corneal and anterior eye segment OCT (PS-CAS-OCT) in visualizing the trabecular meshwork (TM) based on statistical evidences. A total of 31 normal subjects participated in this study. The anterior eye segments of both the eyes of the subjects are scanned using a custom-made PS-CAS-OCT and the standard-scattering OCT (S-OCT) and polarizationsensitive phase-retardation OCT (P-OCT) images are obtained. Three graders grade the visibility of the TM using a four-leveled grading system. The intergrader agreement, intermodality differences, and interquadrant dependence of visibility are statistically examined. All three of three combinations of graders show substantial agreement in visibility with P-OCT ( $\rho=0.74,0.70$, and 0.68 , Spearman's correlation), while only one of three shows substantial agreement with S-OCT $(\rho=0.72)$. Significant dependence of the visibility on the modality (S-OCT versus P-OCT) and quadrants are found by the analysis of variance. A subsequent Wilcoxon signed-rank test reveals significantly improved visibility. PS-CAS-OCT may become a useful tool for screening angle-closure glaucoma. ( 2010 Society of Photo-Optical Instrumentation Engineers. [DOI: 10.1117/1.3499421]
\end{abstract}

Keywords: optical coherence tomography; polarization; birefringence; ophthalmology; anterior eye segment; glaucoma.

Paper 10012SSR received Jan. 12, 2010; revised manuscript received May 25, 2010; accepted for publication Jun. 9, 2010; published online Dec. 23, 2010.

\section{Introduction}

Angle-closure glaucoma (ACG) is a type of glaucoma that is triggered by an anatomical disorder in the anterior segment of the eye. At a ciliary body, aqueous humor is generated, introduced to the anterior eye chamber, and drained from the trabecular meshwork. The anatomical blockage of the drainage channel of the trabecular meshwork triggers the elevation of intraocular pressure (IOP), and this elevated IOP damages the optic nerve. ${ }^{1}$ This acute process is referred to as an attack of ACG. Since the prevalence of ACG is high, ${ }^{2-9}$ it is important to assess the risk of ACG before the onset of symptoms by using suitable screening procedures.

In a conventional screening procedure, the opening of the iridocorneal angle is assessed by a contact gonioscopic lens, and the position of the trabecular meshwork is used as a landmark for the angle assessment. Anterior-eye-segment optical coherence tomography ${ }^{10}$ (AS-OCT) and 3-dimensional (3-D) cornea-andanterior-eye-segment OCT (3-D CAS-OCT) (Refs. 11 and 12) 
are recent technologies that provide tomographic images of the cross section of an anterior eye segment in a noncontact manner. Since this equipment can be easily operated and is comfortable for the patients, it has been widely utilized for ACG screening. By using compatibility between the image properties of OCT and ultrasound, ultrasound-based structural parameters, such as angle-opening distance at $500 \mu \mathrm{m}$ (AOD500) (Ref. 13) and angle recess area ${ }^{14}$ (ARA) have been applied to perform the OCT-based screening. Since the trabecular meshwork is rarely visible in the OCT images, these parameters are assessed using the location of a scleral spur as a landmark.

Occasionally, although the OCT screening protocol may have been properly employed, the scleral spur may not be visible in the OCT image. A previous study has shown that the scleral spur is visible in only $72 \%$ of the OCT images, ${ }^{15}$ i.e., the mentioned parameters are not applicable to more than a quarter of the general population. The use of the trabecular meshwork as a landmark may be more appropriate not only because of the visibility issue of the scleral spur but also because the glaucoma attack is triggered by an anatomical blocking of the trabecular meshwork.

The trabecular meshwork appears as a diffuse hyperscattering region in an OCT image, ${ }^{10-12}$ but it is not always clearly visualized. Therefore, it is important to establish new types of OCT with improved contrast mechanisms that can be used to clearly visualize the trabecular meshwork.

The trabecular meshwork is a collagenous tissue with a perforate structure. ${ }^{16}$ Consequently, the meshwork shows birefringence, and it can be selectively visualized ${ }^{17,18}$ by using a polarization-sensitive extension of OCT. This modified OCT, referred to as polarization-sensitive OCT (PS-OCT), is an OCT system that measures the polarization phase retardation caused by the birefringence of a sample. ${ }^{19,20}$ PS-OCT can simultaneously provide phase-retardation tomography (P-OCT) and standard scattering OCT (S-OCT) images. This new type of OCT has been used for the investigation of the posterior ${ }^{21-25}$ and anterior eye segments, ${ }^{26}$ quantitative assessment of a retinal nerve fiber layer, ${ }^{27-30}$ and follow-up investigations after glaucoma surgery. ${ }^{31}$

Although it is known that PS-OCT has the potential to clearly visualize the trabecular meshwork, there are no evidence-based studies for proving this improved visibility. ${ }^{17,18}$ In this paper, we aim to provide, to the best of our knowledge, the first statistical evidence of the enhanced visibility of the trabecular meshwork by PS-OCT.

\section{Methods}

\subsection{Subjects and Protocol}

We recruited 31 subjects who did not show any marked disorder in the anterior eye segment. The subjects, who were recruited at the University of Tsukuba, consisted of 24 men, and 7 women, and the mean age of the subjects was $26.6 \pm 6.0 \mathrm{yr}$ (mean \pm standard deviation; ranged from 22 to $48 \mathrm{yrs}$ ).

The IOPs of both eyes of all the subjects were measured by using a Goldman applanation tonometer. After the tonometric examination, the subjects were examined ${ }^{18}$ using our 3-D PSCAS-OCT. The tilting angle of the eye was minimized as the angle between the bottom line of the iris becomes parallel to the bottom line of the OCT image. During the 3-D PS-OCT scan, which typically takes $3.3 \mathrm{~s}$, the eye was kept open by using a speculum. Since the tonometric examination and the eye speculum were contact methods, $0.4 \%$ topical oxybuprocaine was applied to anesthetize the cornea. The scanning protocol involved a horizontal fast-raster scan with $256 \times 256 \mathrm{~A}$-lines, and the transversal scanning area was $16 \times 16 \mathrm{~mm}$. The PSOCT examination was performed on both eyes. Thus, a total of 62 eyes were examined. Before and after the OCT scan, the integrity of the eye was examined by a standard slit-lamp microscope.

The study protocol, purpose of the study, and the potential risks of the examination were explained to the subjects orally, and in writing, and written informed consent was obtained from all of the subjects.

All the examinations conformed to the Declaration of Helsinki, and all the protocols were approved by the Institutional Review Board of the University of Tsukuba.

\section{$2.2 \quad P S-O C T$}

A custom-built 3-D PS-CAS-OCT was employed in this study. This PS-OCT is an extension of our 3-D swept-source CASOCT (Ref. 11), and it can acquire 3-D S-OCT and P-OCT images simultaneously. Since the details of the system are comprehensively described in Ref. 18, we provide only a brief summary of the working principle of this instrument.

This PS-OCT continuously modulates the polarization state of the probe beam along the wavelength tuning of frequencydomain optical detection. Consequently, the probe beam possesses two orthogonal polarization components; one of these components is modulated in its phase, and the other is not modulated. The probe beam illuminates the sample, is scattered back, introduced to a polarization-sensitive photodetector pair, and forms a pair of spectral-interference fringes as a result of the interference with a reference beam. One of the detectors of the photodetector pair is sensitive to the horizontal polarization state (horizontal channel), and the other is sensitive to the vertical polarization state (vertical channel). Since the polarization axes of the polarization-sensitive detectors were not identical to the polarization axes of the already-mentioned modulation, we obtained four combinations of the polarization states: modulated in phase and detected by the horizontal channel, modulatedvertical, nonmodulated-horizontal, and nonmodulated-vertical. These four combinations of polarization yield four complex OCT images, which are processed using a custom-made PSOCT algorithm to yield a depth-resolved Jones-matrix tomography. Each Jones matrix of the tomography is then diagonalized and resolved to physically meaningful parameters: phase retardation (the relative phase between the eigenvalues of the Jones matrix), diattenuation coefficients (the ratio of squared amplitudes of the eigenvalues), and optic axis orientation (the direction of the eigenvectors). The depth-resolved phase-retardation map obtained is the final PS-OCT image, which is referred to as a P-OCT image. Further, the intensities of the nonmodulated complex OCT images are averaged to provide an S-OCT image.

The center wavelength of PS-OCT was $1.31 \mu \mathrm{m}$, the depth and transversal optical resolutions were $11.9 \mu \mathrm{m}$ (in air) and $32.2 \mu \mathrm{m}$, respectively, and the acquisition speed was 20,000 A-lines/s. The scanning protocol utilized in this particular study provided the transversal $A$-line spacing of $62.5 \mu \mathrm{m}$, 


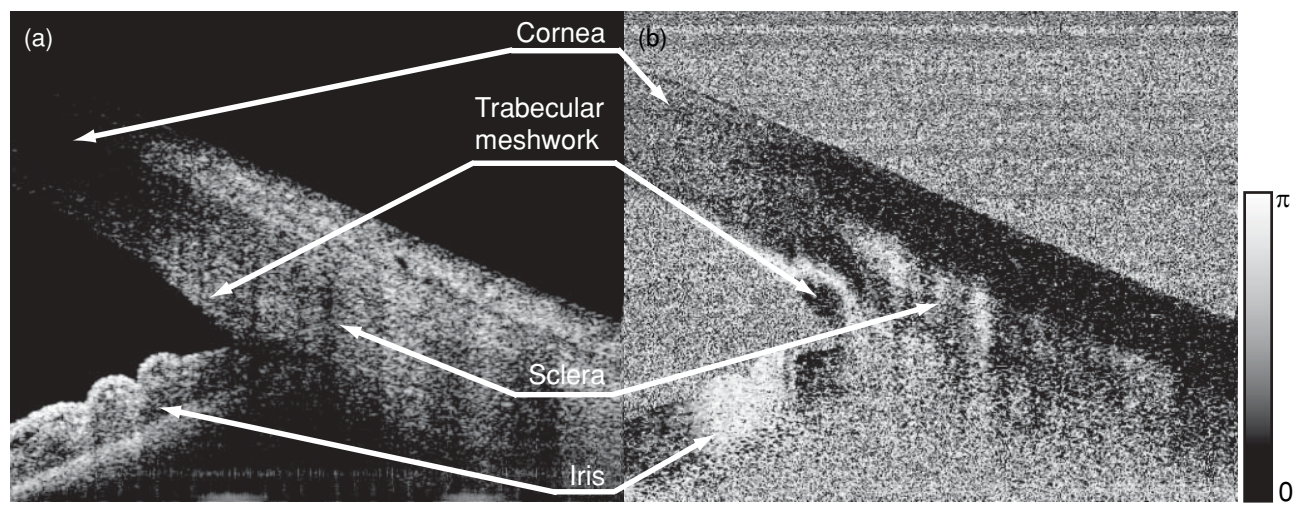

Fig. 1 Examples of (a) S-OCT and (b) P-OCT images of human anterior eye angle. The trabecular meshwork appears as a diffusive hyper scattering region in S-OCT, while it appears as a bending fringe pattern in P-OCT. These images are created from Interactive Scientific Publishing (ISP) data sets of Ref. 31.

and hence this $A$-line spacing dominated the nominal transversal resolution. During the scanning procedure, the optical power incident on the cornea was $4.0 \mathrm{~mW}$, which satisfied the safety standard of ANSI (Ref. 32).

The examples of P- and S-OCT images of an anterior eye segment are shown in Fig. 1. In the S-OCT image [Fig. 1(a)], the trabecular meshwork appears as a diffusive scattering region, while it appears as a bending fringe pattern in the corresponding P-OCT image [Fig. 1(b)]. This is caused by a characteristic birefringence property of the trabecular meshwork, and this fringe pattern makes the recognition of the trabecular meshwork easy. The detailed mechanism of this appearance is discussed in Sec. 4, and the superior visibility of the trabecular meshwork by $\mathrm{P}-\mathrm{OCT}$ to $\mathrm{S}-\mathrm{OCT}$ is discussed in the following sections.

\subsection{Image Preparation and Grading}

After the mentioned PS-OCT examinations, the S- and P-OCT images were processed using the following protocol. From each 3-D OCT volume, images corresponding to four virtual cross sections, namely, the temporal, superior, nasal, and inferior quadrants were extracted. The images were properly flipped to align their angle corners to the left side of the image. This step was performed to blind the quadrant from the graders who subjectively graded the visibility of the trabecular meshwork. Since four S-OCT images and four P-OCT images were created from each eye, a total of 496 images were used in the subsequent grading process.

All the quadrant images were then examined by three graders, including two experienced ophthalmologists, KK and SF, who used CAS-OCT (Visante OCT, Carl Zeiss Meditec, California) in their daily clinical routines. The third grader was an OCT engineer, YY, who is experienced in both standard OCT and PS-OCT. The quadrant images were presented to the graders in a random sequence, and each grader was blinded to the results provided by the other graders. The graders graded the visibility of the trabecular meshwork in the S- and P-OCT images on the basis of the grading criteria presented in Table 1. This criteria were designed not to directly evaluate an image contrast but to evaluate the clinical significance of OCT images. This grading concept enabled a fair comparison of P-OCT and S-OCT, which were based on different imaging mechanisms. This visibility defined here was referred to as the score visibility. Figure 2 shows the examples of the S- and P-OCT images corresponding to each score.

A binary visibility was then determined by using the score visibility. In the binary visibility, the images with scores of 1 and 2 were labeled as invisible, while images with scores of 3 and 4 were labeled as visible. Then, the sensitivity was defined as the ratio of the number of visible images to the total number of images. The images with scores of 0 (image not available) were excluded from the subsequent analyses.

The cohort was then resized according to the score visibility. Only the subjects with the score visibility better than or equal to 1 for 6 quadrants, i.e., the temporal, nasal, and inferior quadrants of the 2 eyes, were included in the subsequent statistical analysis. A few images presented the score visibility of 0 at these three quadrants mainly because of unoptimized alignment of the eye. The superior quadrants were not used in the analysis, because the images from almost all the subjects showed vignetting caused by the eyelid. After this resizing procedure, 52 eyes from 26 subjects were finally included in the study.

\subsection{Statistical Analysis}

The properties of the score visibility, binary visibility and sensitivity were analyzed by the following statistical methods. The overall property of the score visibility was examined by analysis of variance (ANOVA). The intergrader agreement of the score visibility was analyzed by Spearman's rank correlation. The intermodality differences between the score visibilities for the S- and P-OCT images were examined by Wilcoxon's signed

Table 1 The grading criteria to access the visibility of the trabecular meshwork.

4

Clearly Visible, and the location is identified

3

Visible

2

Moderately Visible, but could be artifact

1

Invisible

0

Image not available 

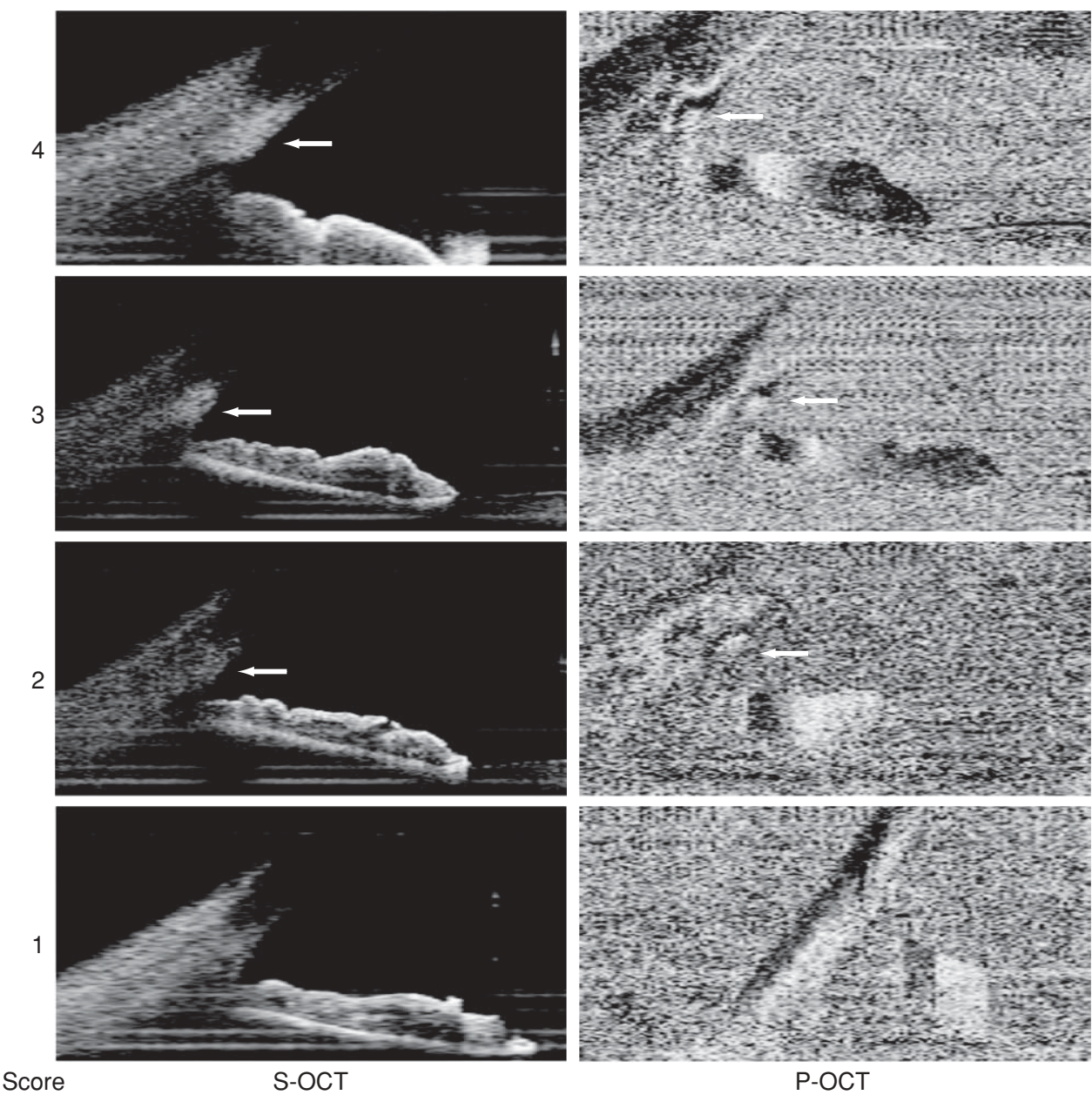

Fig. 2 Examples of S- and P-OCT images with each grading score. The arrows indicate the possible locations of trabecular meshwork. The larger scores indicate a higher visibility of the trabecular meshwork. Here the respective S- and P-OCT images for the same score are not necessarily created from the same data set.

rank test. The interquadrant dependence of the score visibility was examined by Wilcoxon's signed-rank test. For these analyses of score visibility, rank correlation and rank test were used because the score visibility was represented by discrete ranks.

The overall properties of the binary visibility were analyzed by logistic regression analysis, while the intergrader agreement was examined by Fleiss's kappa test. The intermodality differences and the interquadrant dependence of the sensitivity were examined by Fisher's exact test. For these analyses of binary visibility, we properly selected the statistics suitable for the analysis of binary data.

Both for the score visibility and binary visibility the mentioned statistical analyses were performed for the following purposes. Intergrader agreements were examined to evaluate the consistency of grading results among the three graders. This consistency may indicate grading objectivity. The intermodality differences were examined to prove the superior performance of $\mathrm{P}-\mathrm{OCT}$ to S-OCT. The interquadrant analyses were performed to reveal the further direction-dependent contrast property of $\mathrm{S}-\mathrm{OCT}$ and P-OCT.

The left eyes were used for analyzing intermodality and interquadrant differences in the score visibility, while the right eyes were used for analyzing intermodality and interquadrant differences in sensitivity.

The statistical analyses were performed by using the statistics computing language $\mathrm{R}$. Most of the statistical analyses were performed using a base-package of $\mathrm{R}$, and additional libraries, including Coin-package, were properly used to calculate the tie-corrected exact $p$ values.

\section{Results}

\subsection{Score Visibility}

The overall properties of the score visibilities recorded by the three graders were examined by ANOVA where the explaining variables were the type of OCT (S- and P-OCT, denoted as modality), quadrant, laterality, IOP, and age. All interactions of two of the explaining variables were accounted. The results of this analysis are summarized in Table 2. For all the three graders, the score visibilities showed statistically significant dependence on the modality (S- and P-OCT) and the quadrants, and there was a significant interaction between the modality and quadrant in the scores recorded by all the three graders.

The intergrader agreements for the score visibilities were evaluated for both S- and P-OCT images. We observed a 
Table 2 ANOVA $p$ values of score visibilities.

\begin{tabular}{llll}
\hline Factor & KK & SF & YY \\
\hline Modality & $<0.001^{* *}$ & $<0.001^{* *}$ & $<0.001^{* *}$ \\
Quadrant & $<0.001^{* *}$ & $<0.001^{* *}$ & $<0.001^{* *}$ \\
Laterality & 0.20 & 0.36 & 0.73 \\
IOP & 0.16 & 0.83 & 0.27 \\
Age & 0.65 & 0.77 & 0.78 \\
Modality-quadrant & $0.020^{*}$ & $0.020^{*}$ & $0.0013^{* *}$ \\
Modality-laterality & 0.11 & 0.88 & 0.84 \\
Modality-IOP & 0.42 & 0.92 & 0.22 \\
Modality-age & 0.98 & 0.056 & 0.84 \\
Quadrant-laterality & 0.68 & 0.25 & 0.94 \\
Quadrant-IOP & 0.12 & 0.84 & 0.91 \\
Quadrant-age & 0.11 & 0.48 & 0.53 \\
Laterality-IOP & 0.25 & 0.98 & 0.35 \\
Laterality-age & 0.22 & 0.36 & 0.20 \\
IOP-age & 0.28 & $0.0036^{* *}$ & 0.20 \\
\hline
\end{tabular}

Each column represent the graders, and the hyphens represents interaction. Modality is the type of OCT (S- and P-OCT). Statistical significance is *at $p<$ 0.05 and ** at $p<0.01$.

substantial agreement between the S-OCT-based scores in one of the three combinations of graders $(\rho=0.72, p<0.0001)$, and the remaining two combinations showed moderate agreements $(\rho=0.58$ and $0.59, p<0.0001$ for both), where $\rho>0.60$ was regarded as substantial. For the P-OCT-based scores, all the three combinations of graders showed substantial agreement ( $\rho=0.74,0.68$, and $0.70, p<0.0001$ for all).

The average scores of the score visibility for each modality, and each grader are summarized in Fig. 3 Since we did not observe any significant dependence on laterality in the ANOVA, only the left eyes were analyzed. The average scores for the P-OCT images were better than those for the S-OCT images, for all quadrants and all graders. Statistically significant differences existed between the temporal-quadrant scores recorded by all the graders.

The interquadrant differences in the scores for the left eyes were analyzed by a Wilcoxon signed-rank test. The mean score differences for each combination of quadrants are summarized in Table 3. For all the graders and both modalities, the scores for the inferior quadrant were significantly lower than those for the temporal and nasal quadrants. The average scores for the nasal quadrant were better than those for the temporal quadrant for all the graders, and was statistically significant for YY. In contrast, the average P-OCT scores for the temporal quadrant were better than those for the nasal quadrant, for all the graders.

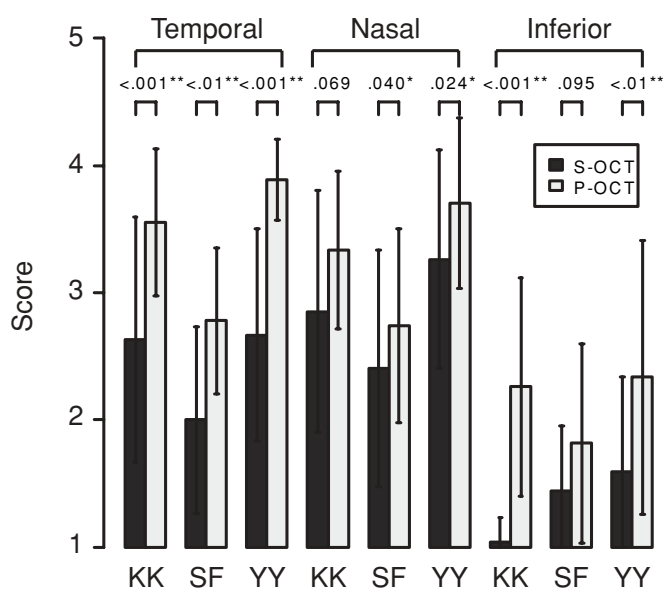

Fig. 3 Average scores of left eye. The values over the bars are the $p$ values of the Wilcoxon signed-rank test between S- and P-OCT scores. The whiskers indicate the standard deviations.

\subsection{Binary Visibility and Sensitivity}

The average sensitivities of S- and P-OCT were 40.7 and $65.8 \%$, respectively, where the average sensitivities are the averages of the three quadrants and the three graders at right eyes. The overall properties of the binary visibility, and therefore the sensitivity, were analyzed by logistic regression analysis, as summarized in Table 4 . The binary visibilities recorded by all the three graders were found to be significantly dependent on the OCT modality. We also found a statistically significant interaction between the nasal and inferior quadrants for all three graders.

The intergrader agreement for the sensitivity values was evaluated by using the Fleiss kappa statistics. The Fleiss kappa values were 0.529 for S-OCT and 0.534 for P-OCT. These kappa values indicate moderate intergrader agreement both for S- and P-OCT, which implies that subjective variation among the graders did not strongly affect the sensitivity.

The sensitivities calculated from the scores for the right eyes and their corresponding intermodality differences are summarized in Table 5. For all the three graders, the results showed superior sensitivity of P-OCT to S-OCT. At the inferior quadrant, the improved sensitivity was found by two of three graders. Additional analyses of the left eye showed similar results.

The interquadrant differences in the binary visibility were analyzed by using the dataset of the right eyes in Fisher's exact test. Table 6 shows a summary of the interquadrant difference obtained by a representative grader (KK). The residual two graders showed similar results. For all three of three graders and for both S- and P-OCT, statistically significant interquadrant differences were found for temporal-nasal and nasal-inferior quadrants. Although we did not find any statistical significance, note that the odds ratios of S-OCT for the temporal-nasal quadrant were less than unity for all the graders, while the corresponding odds ratios for the P-OCT images were greater than unity for all the graders. This suggests that S-OCT has better visibility at the nasal quadrant than the temporal quadrant, while P-OCT has better visibility at the temporal quadrant than the nasal quadrant. 
Yasuno et al.: Visibility of trabecular meshwork by standard and polarization-sensitive ...

Table 3 The mean differences of score-visibility for each combination of two quadrants.

\begin{tabular}{ccccc}
\hline & KK & SF & YY \\
\hline S-OCT & $\mathrm{T}-\mathrm{N}$ & $-0.22(0.35)$ & $-0.41(0.10)$ & $-0.59(0.01)$ \\
& $\mathrm{T}-\mathrm{I}$ & $1.59\left(<0.001^{* *}\right)$ & $0.56\left(<0.01^{* *}\right)$ & $1.07\left(<0.001^{* *}\right)$ \\
& $\mathrm{N}-\mathrm{I}$ & $1.81\left(<0.001^{* *}\right)$ & $0.96\left(<0.001^{* *}\right)$ & $1.67\left(<0.001^{* *}\right)$ \\
P-OCT & $\mathrm{T}-\mathrm{N}$ & $0.22(0.21)$ & $0.04(0.87)$ & $0.19(0.36)$ \\
& $\mathrm{T}-\mathrm{I}$ & $1.30\left(<0.001^{* *}\right)$ & $0.96\left(<0.001^{* *}\right)$ & $1.56\left(<0.001^{* *}\right)$ \\
& $\mathrm{N}-\mathrm{I}$ & $1.07\left(<0.001^{* *}\right)$ & $0.93\left(<0.001^{* *}\right)$ & $1.37\left(<0.001^{* *}\right)$ \\
\hline
\end{tabular}

The values are represented by the mean difference ( $p$ value). $T, N$, and I indicate temporal, nasal, and inferior quadrants. Quadrant 1 to quadrant 2 represents the subtraction of the score of quadrant 2 from that of quadrant 1 . * ${ }^{*}$ Statistical significance at $p<0.01$.

\section{Discussion and Conclusion}

The results of the intergrader agreement analysis of the binary visibility for the P-OCT images showed a comparable degree of agreement with those for the S-OCT images. In contrast, in the intergrader analysis of the visibility scores, we found that the number of grader combinations showing substantial agreement for the P-OCT-based assessments was greater than the corresponding number for the S-OCT-based assessments. Since the agreement for binary visibility assessments is not affected by the score fluctuation between 1 and 2, and between 3 and 4, the results imply that P-OCT has a higher intergrader agreement, especially between scores 1 and 2, and scores 3, and 4.

The ANOVA of the score visibility (Table 2) and the logisticregression analysis of the binary visibility (Table 4) indicated a significant improvement in the visibility when using P-OCT. Subsequent analyses with Wilcoxon signed-rank test (Fig. 3), and Fisher's exact test (Table 5) of the intermodality difference showed that there was a significant improvement in the temporal quadrant visibility for all the graders when using P-OCT. In the same analysis, two of the three graders showed significantly improved visibility when analyzing inferior quadrant P-OCT images. At the nasal quadrant, the average score and the sensi-

Table 4 The overall properties of the binary-visibility.

\begin{tabular}{lccc}
\hline Factor & KK & SF & YY \\
\hline Modality & $<0.001^{* *}$ & $<0.001^{* *}$ & $<0.001^{* *}$ \\
Quadrant (T) & 0.071 & $0.026^{*}$ & 0.47 \\
Quadrant (I) & $<0.001^{* *}$ & $<0.001^{* *}$ & $<0.001^{* *}$ \\
Laterality & 0.99 & 0.65 & 0.60 \\
IOP & 0.75 & 0.89 & 0.78 \\
Age & 0.25 & 0.65 & 0.13 \\
\hline
\end{tabular}

The values are $p$ values of logistic regression analysis. Modality is a binary valuable that takes S-OCT or P-OCT. T and I indicate temporal and inferior quadrant. For this quadrant analysis, the sensitivity at the nasal quadrant was used as a reference. Statistical significance is *at $p<0.05$ and ** at $p<0.01$. tivity of P-OCT were higher than those of S-OCT, although the statistical significance was low.

Although there are some reports of preliminary single cases in which the use of PS-OCT improved the contrast of the trabecular meshwork, ${ }^{17,18}$ to the best of our knowledge, this is the first statistical-evidence-based study to prove the enhanced visibility afforded by by PS-OCT for visualizing the trabecular meshwork.

The improved visibility can be attributed to the differences in the appearance of the trabecular meshwork in S- and P-OCT images. In S-OCT images, the trabecular meshwork appears as a diffuse hyperscattering region. To identify the trabecular meshwork in S-OCT images, an observer is required to identify a relative alteration of the OCT intensity, which is occasionally difficult to determine. In contrast, in P-OCT, the trabecular meshwork appears as a bending fringe pattern, since the trabecular meshwork is a collagenous tissue, and possesses a characteristic birefringence, and P-OCT is sensitive to tissue birefringence. P-OCT visualizes the phase retardation induced by the tissue birefringence and accumulated along the depth of the tissue. Therefore, tissues with strong birefringence present a fringe pattern in the P-OCT image, and this pattern alters along the depth of the tissue. It is often found that in cross-sectional P-OCT, a 2-D fringe pattern is bent around a localized birefringent tissue. Note here that this bending of the fringe does not mean bending of a structure, but a nonuniform cumulative phase retardation of the OCT probe beam. Similar hypercumulative phase retardation at a point does not necessarily mean the hyperbirefringence at that point. However, it has been known that, in particular, a bending fringe is formed at the trabecular meshwork. ${ }^{17,18}$ To identify the trabecular meshwork in P-OCT images, the observer is required to identify a deformation of a clearly visualized fringe pattern. Such deformations are easier to detect and can be more qualitative than the already mentioned intensity alteration. These properties of P-OCT may explain the higher intergrader agreement and the improved visibility.

An ANOVA of the score visibility (Table 2) as well as the logistic-regression analysis of the binary visibility assessments (Table 4) showed significant interquadrant dependence. Subsequent Wilcoxon signed-rank tests using the score visibility (Table 3) and Fisher's exact tests using the binary visibility (Table 6) showed that there were significant differences 
Yasuno et al.: Visibility of trabecular meshwork by standard and polarization-sensitive ...

Table 5 The summary of sensitivities.

\begin{tabular}{|c|c|c|c|c|c|c|c|c|c|c|}
\hline \multirow[b]{2}{*}{ Grader } & \multirow[b]{2}{*}{ Modality } & \multicolumn{3}{|c|}{ Temporal } & \multicolumn{3}{|c|}{ Nasal } & \multicolumn{3}{|c|}{ Inferior } \\
\hline & & Visible & $(\%)$ & $p$ value & Visible & $(\%)$ & $p$ value & Visible & $(\%)$ & $p$ value \\
\hline \multirow[t]{3}{*}{ KK } & S-OCT & 15 & $(55.6)$ & ** & 7 & (25.9) & & 1 & (3.7) & * \\
\hline & & & & 0.0041 & & & 0.25 & & & 0.050 \\
\hline & P-OCT & 25 & $(92.6)$ & & 19 & (70.3) & & 7 & (25.9) & \\
\hline \multirow[t]{3}{*}{ SF } & S-OCT & 10 & (37.0) & * & 12 & (44.4) & & 1 & (3.7) & \\
\hline & & & & $0.028^{*}$ & & & 0.17 & & & 0.35 \\
\hline & P-OCT & 19 & (70.4) & & 18 & (66.7) & & 4 & (14.8) & \\
\hline \multirow[t]{3}{*}{ YY } & S-OCT & 14 & (51.9) & & 22 & (81.5) & & 3 & (1) 1.1$)$ & \\
\hline & & & & $0.0003 * *$ & & & 0.42 & & & 0.028 * \\
\hline & P-OCT & 26 & (96.3) & & 25 & (92.6) & & 11 & $(40.7)$ & \\
\hline
\end{tabular}

The "Visible" columns show the numbers of quadrant-images labeled as visible in the binary visibility. The $p$ values are Fisher's exact $p$ values. Statistical significance is * at $p<0.05$ and ${ }^{* *}$ at $p<0.01$.

between the visibilities in the inferior quadrant and the other two quadrants in both S- and P-OCT images. This difference may be attributed to the scanning protocol. The images corresponding to the temporal and nasal quadrants were obtained along the fast-scanning direction of the raster scanning protocol, and the corresponding acquisition time was $13 \mathrm{~ms}$; in contrast, the inferior quadrant image was obtained along the slow-scanning direction, and the corresponding acquisition time was $3.3 \mathrm{~s}$. This difference in acquisition times resulted in differences in the effects of subject motion and OCT-phase stability, consequently affecting the image quality. A cross-scanning protocol, in which horizontal temporal-nasal scan was followed by vertical superior-inferior scan, could reduce this possibly artificial differences of visibilities.

Although its statistical significance was low, we found another interesting tendency of the visibility scores in the interquadrant analysis (Table 3). In S-OCT images, the average

Table $\mathbf{6}$ Interquadrant difference in sensitivity obtained by a representative grader (KK).

\begin{tabular}{lccc}
\hline & Odds Ratio & $p$ Value \\
\hline S-OCT & T-N & 0.36 & 0.15 \\
& $T-1$ & 30.41 & $<0.001$ ** \\
& $\mathrm{N}-\mathrm{I}$ & 80.22 & $<0.001$ ** \\
P-OCT & $\mathrm{T}-\mathrm{N}$ & 1 & 1.0 \\
& $\mathrm{~T}-\mathrm{I}$ & 32.50 & $<0.001$ ** \\
& $\mathrm{N}-\mathrm{I}$ & 32.50 & $<0.001$ ** \\
\hline
\end{tabular}

The $p$ values are Fisher's exact $p$ values. At the "quadrant 1 - quadrant 2," row an odds ratio larger than unity indicates that the sensitivity of quadrant 1 is better than quadrant 2 and vice versa. ${ }^{*}$ Statistical significance at $p<0.01$. nasal quadrant scores of score visibility recorded by all the graders were higher than the corresponding scores for the temporal quadrant; in contrast, in the P-OCT images, the average temporal quadrant scores recorded by all the graders were higher than the corresponding scores for the nasal quadrant. We observed the same tendency in the sensitivity values (Table 6). Note here that the interquadrant analysis of the visibility scores was performed for the data set of the left eyes, while that of the sensitivity values was performed for the data set of the right eyes; therefore, these two results are fairly independent of each other. The already mentioned differences in scanning direction cannot explain this interquadrant dependence, because the nasal, and temporal quadrants were simultaneously scanned along the fast-scanning direction. We hypothesized three reasons, i.e., system asymmetricity, tilting of the eye, and tissue asymmetricity, to explain this interquadrant dependence.

The first scenario is based on a system asymmetricity associated with the scanning direction of the fast horizontal scan. Our PS-OCT system always scans the eye from the 3- to the 9-o'clock position, and this fixed scanning pattern could have introduced bias in the visibility. If this hypothesis was valid, the nasal-temporal tendencies between the left and right eyes would be opposite of each other, because the scanning is performed from the temporal to the nasal quadrant for the left eyes and from the nasal to the temporal quadrant for the right eyes. However, additional analyses of the score visibility and binary visibility of the eyes by interchanging the sides showed the same tendency observed in the previous analyses, i.e., the nasal quadrant was better for S-OCT, and the temporal quadrant was better for P-OCT. Therefore, we rejected this hypothesis.

The next scenario involved the relative horizontal tilt angle between the eye and the probe beam. The tilt angle of the eye may possess a systematic bias, because the human eye is tilted slightly inward while observing an object. In addition to this possible tilting, if the trabecular meshwork possesses anisotropic light-scattering properties, the tilting can induce interquadrant 

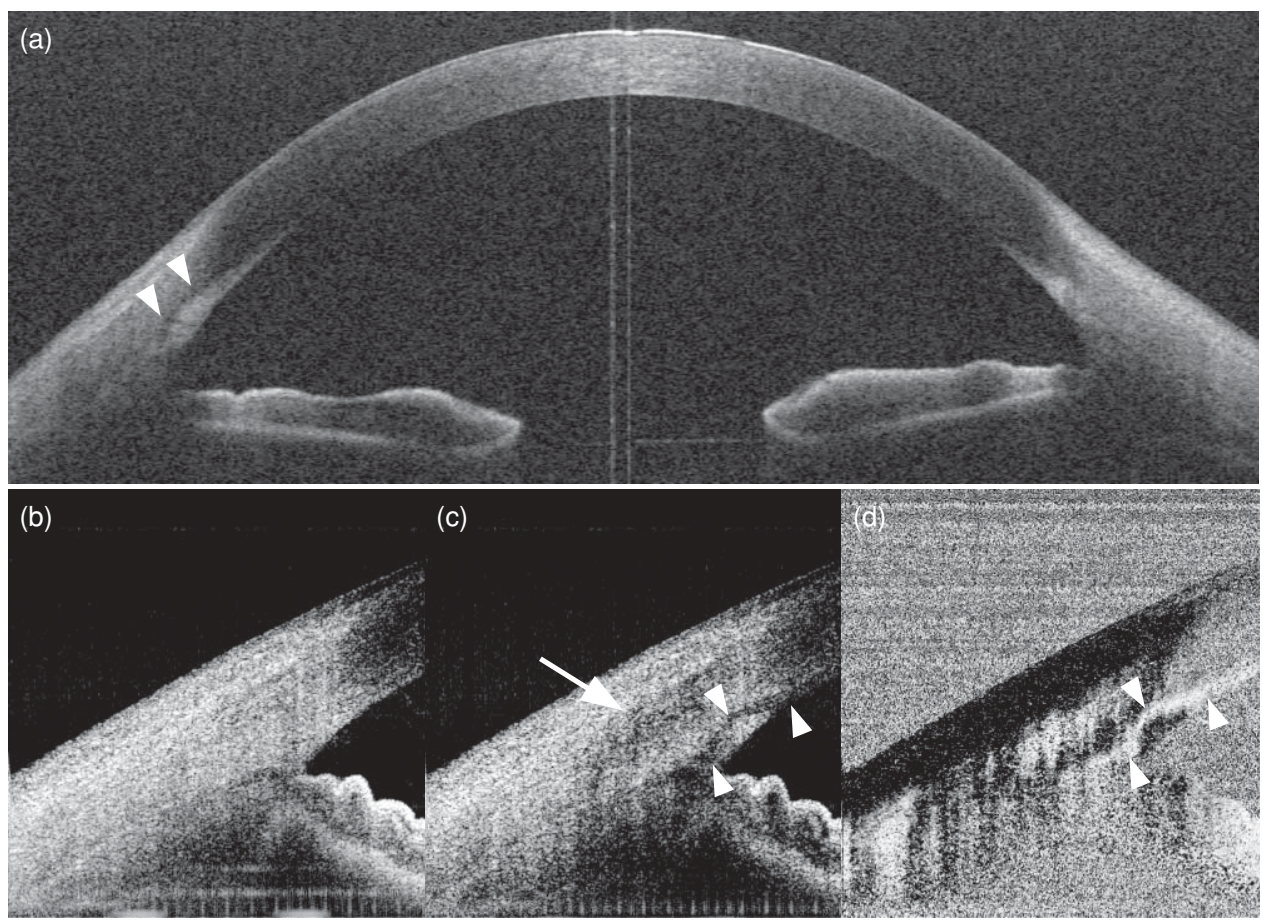

Fig. 4 Example of birefringence artifact at the angle. (a) Example of an anterior eye segment tomography taken by commercially available CAS-OCT (CASIA, Tomey Corp., Aichi, Japan), where the arrowheads indicate a dark band surrounding the trabecular meshwork; (b) polarization-independent intensity OCT, which is created from two channels of polarization sensitive detector of PS-OCT; (c) polarization-dependent intensity OCT, which is created from a single detection channel; and (d) the corresponding P-OCT. (b), (c), and (d) are created from the same set of complex OCT signals, which is available as an ISP data set of Ref. 31.

differences. To examine this hypothesis, we measured the tilt angle directly from the OCT images, where the tilt angle of the line that connects the angle recesses with reference to the basal line of the OCT image was regarded as the tilt angle. The correlation between the tilt angle and the visibility score was examined by Spearman's rank-correlation test. However, there was no significant correlation between the scores recorded by the three graders, and this second scenario was fairly rejected.

The final scenario is based on the anisotropy of the tissue. A possible anisotropic optical property of the trabecular meshwork along the tangential angle may explain the interquadrant differences. However, to the best of our knowledge, there have been no studies on the optical properties of the trabecular meshwork. Since the optical property of the tissue is frequently associated with the microstructure of the tissue in the wavelength and subwavelength domains, a study of the optical property of the trabecular meshwork would be important.

The detection of Schlemm's canal is an important topic for anterior eye OCT (Refs. 12 and 33). A dark band structure surrounding the trabecular meshwork was infrequently detected in the conventional scattering-based AS- and CAS-OCT images, as exemplified in Fig. 4(a) (arrowheads); this structure was sometimes regarded as a Schlemm's canal. ${ }^{11,12}$ In this study, we found that the bending fringe pattern of the trabecular meshwork in P-OCT images was similar in its shape to the dark band structure in a conventional OCT image. In principle, a standard OCT does not have any polarization sensitivity. However, it is known that the OCT signal intensity is altered by the relative difference in the polarization states of the reference and probe beams, and this intensity alteration creates an artifact. The similarity of the bending pattern and the abnormal polarization sensitivity of OCT suggest that the dark band structure could be an artifact arising from the birefringence of the trabecular meshwork.

To examine this hypothesis, we created two types of intensity OCT images. One image was obtained by averaging the intensities of OCT images detected by two PS-OCT polarization channels, while the other image was created from a single detection channel. The first type of image is known to be polarization independent, while the latter has the mentioned unauthorized polarization sensitivity and is affected by sample birefringence. Figure 4(b) is an example of the polarizationindependent OCT image, in which no dark band structure was observed. Figure 4(c) is the corresponding intensity OCT image constructed from a single detection channel, in which a dark band structure surrounding the trabecular meshwork was clearly observed (indicated by arrowheads). Further, the structure of the dark band is found to clearly correlate with the band structure in the corresponding P-OCT image [Fig. 4(d)]. On the basis of this examination, we concluded that the dark band surrounding the trabecular meshwork does not always represent the actual structure of Schlemm's canal; it may occasionally indicate an artifact arising from the sample birefringence.

An additional artifact observed in Fig. 4(c) is a hyposcattering region in the sclera, which is indicated by an arrow. Since this hyposcattering region is not observed in the polarizationindependent OCT image [Fig. 4(b)], we assumed that it is an artifact generated by the birefringence of the sclera.

The mean sensitivities of trabecular meshwork were $40.7 \%$ for S-OCT, and $65.8 \%$ for P-OCT, while it was reported ${ }^{15}$ that the sensitivity of scleral spur was found to be $72 \%$ with a 
standard anterior eye segment OCT (Visante OCT). Despite this superior visibility of scleral super by the standard OCT, PS-OCT still possesses advantages over standard OCT because of the following reasons. First, as mentioned, the visibility of sclera spur was reported to be $72 \%$. This indicates the scleral spur is still not detectable for more than a quarter of the population. Additional contrast of trabecular meshwork provided by P-OCT can further improve the accuracy of the assessment of anterior angle. Second, the trabecular meshwork is a drainage channel of aqueous humor and hence is a more direct landmark for the assessment of angle closure than the scleral spur. Finally, however, the most significant advantage is that the PS-OCT simultaneously acquires P-OCT and S-OCT images. Because of this property, any angle assessment techniques that have been applied to the standard OCT are compatible with PS-OCT. Namely, PS-OCT provides additional information without any loss of information. Therefore, the angle assessment by PS-OCT has advantages over that by the standard OCT.

We recently demonstrated PS-OCT-based tissue contrast tomography. ${ }^{34}$ Using this tissue contrast tomography, we can selectively contrast the location of the trabecular meshwork. This may enable automatic identification of the location of the trabecular meshwork, and consequently enable fully automated assessment of the anterior angle in the future.

In conclusion, we compared the visibilities afforded by scattering and phase retardation OCT in visualizing the trabecular meshwork. The statistical analyses showed a significant improvement of visibility in the P-OCT images. This improved visibility may enable structural parameters that can more directly assess the risk of ACG than current methods.

\section{Acknowledgment}

This study was supported by the Japan Science and Technology Agency through the contract of Development of Systems and Technology for Advanced Measurement and Analysis. Financial support from Tomey corporation is gratefully acknowledged.

\section{Financial Discloser}

Grant support was provided by Tomey Corporation to Yasuno, Yamanari, and Makita. Sakai had some employment with Kanebo Cosmetics. Kawana, Miura, Fukuda, and Oshika have no conflicts of interest.

\section{References}

1. R. R. Allingham, K. F. Damji, S. Freedman, S. E. Moroi, and G. Shafranov, Shields' Textbook of Glaucoma, 5th ed., Lippincott Williams \& Wilkins, New York (2004).

2. Y. Shiose, Y. Kitazawa, S. Tsukahara, T. Akamatsu, K. Mizokami, R. Futa, H. Katsushima, and H. Kosaki, "Epidemiology of glaucoma in japan-a nationwide glaucoma survey," Jpn. J. Ophthalmol. 35(2), 133-155 (1991)

3. P. J. Foster, J. Baasanhu, P. H. Alsbirk, D. Munkhbayar, D. Uranchimeg, and G. J. Johnson, "Glaucoma in mongolia. a population-based survey in hövsgöl province, northern mongolia," Arch. Ophthalmol. 114(10), 1235-1241 (1996).

4. C. Cedrone, F. Culasso, M. Cesareo, A. Zapelloni, P. Cedrone, and L. Cerulli, "Prevalence of glaucoma in Ponza, Italy: a comparison with other studies," Ophthalmic Epidemiol. 4(2), 59-72 (1997).
5. M. D. Wensor, C. A. McCarty, Y. L. Stanislavsky, P. M. Livingston, and H. R. Taylor, "The prevalence of glaucoma in the melbourne visual impairment project," Ophthalmology 105(4), 733-739 (1998).

6. L. Bonomi, G. Marchini, M. Marraffa, P. Bernardi, I. D. Franco, S. Perfetti, A. Varotto, and V. Tenna, "Prevalence of glaucoma and intraocular pressure distribution in a defined population. The Egnaneumarkt Study," Ophthalmology 105(2), 209-215 (1998).

7. P. J. Foster, F. T. Oen, D. Machin, T. P. Ng, J. G. Devereux, G. J. Johnson, P. T. Khaw, and S. K. Seah, "The prevalence of glaucoma in chinese residents of singapore: a cross-sectional population survey of the tanjong pagar district," Arch. Ophthalmol. 118(8), 1105-1111 (2000).

8. R. R. Buhrmann, H. A. Quigley, Y. Barron, S. K. West, M. S. Oliva, and B. B. Mmbaga, "Prevalence of glaucoma in a rural east african population," Invest. Ophthalmol. Vis. Sci. 41(1), 40-48 (2000).

9. H. A. Quigley, S. K. West, J. Rodriguez, B. Munoz, R. Klein, and R. Snyder, "The prevalence of glaucoma in a population-based study of hispanic subjects, Proyecto ver," Arch. Ophthalmol. 119(12), 18191826 (2001).

10. S. Radhakrishnan, A. Rollins, J. Roth, S. Y. V. Westphal, D. Bardenstein, and J. Izatt, "Real-time optical coherence tomography of the anterior segment at $1310 \mathrm{~nm}$," Arch. Ophthalmol. 119, 1179-1185 (2001).

11. Y. Yasuno, V. D. Madjarova, S. Makita, M. Akiba, A. Morosawa, C. Chong, T. Sakai, K. Chan, M. Itoh, and T. Yatagai, "Threedimensional and high-speed swept-source optical coherence tomography for in vivo Investigation of human anterior eye segments," Opt. Express 13, 10652-10664 (2005).

12. S. Asrani, M. Sarunic, C. Santiago, and J. Izatt, "Detailed visualization of the anterior segment using fourier-domain optical coherence tomography," Arch. Ophthalmol. 126(6), 765-771 (2008).

13. C. J. Pavlin, K. Harasiewicz, and F. S. Foster, "Ultrasound biomicroscopy of anterior segment structures in normal and glaucomatous eyes," Am. J. Ophthalmol. 113(4), 381-389 (1992).

14. H. Ishikawa, J. M. Liebmann, and R. Ritch, "Quantitative assessment of the anterior segment using ultrasound biomicroscopy," Curr. Opin. Ophthalmol. 11(2), 133-139 (2000).

15. L. M. Sakata, R. Lavanya, D. S. Friedman, H. T. Aung, S. K. Seah, P. J. Foster, and T. Aung, "Assessment of the scleral spur in anterior segment optical coherence tomography images," Arch. Ophthalmol. 126(2), 181-185 (2008).

16. M. J. Hogan, Histology of the Human Eye, Saunders, Philadelphia (1971).

17. M. Pircher, E. Götzinger, R. Leitgeb, and C. K. Hitzenberger, "Transversal phase resolved polarization sensitive optical coherence tomography," Phys. Med. Biol 49(7), 1257-1263 (2004).

18. M. Yamanari, S. Makita, and Y. Yasuno, "Polarization-sensitive sweptsource optical coherence tomography with continuous source polarization modulation," Opt. Express 16(8), 5892-5906 (2008).

19. M. R. Hee, D. Huang, E. A. Swanson, and J. G. Fujimoto, "Polarizationsensitive low-coherence reflectometer for birefringence characterization and ranging," J. Opt. Soc. Am. B 9, 903-908 (1992).

20. J. F. de Boer, T. E. Milner, M. J. C. van Gemert, and J. S. Nelson, "Twodimensional birefringence imaging in biological tissue by polarizationsensitive optical coherence tomography," Opt. Lett. 22, 934-936 (1997).

21. E. Götzinger, M. Pircher, and C. K. Hitzenberger, "High speed spectral domain polarization sensitive optical coherence tomography of the human retina," Opt. Express 12, 10217-10229 (2005).

22. M. Yamanari, S. Makita, V. D. Madjarova, T. Yatagai, and Y. Yasuno, "Fiber-based polarization-sensitive fourier domain optical coherence tomography using b-scan-oriented polarization modulation method," Opt. Express 14, 6502-6515 (2006).

23. M. Pircher, E. Götzinger, O. Findl, S. Michels, W. Geitzenauer, C. Leydolt, U. Schmidt-Erfurth, and C. K. Hitzenberger, "Human macula Investigated in vivo with polarization-sensitive optical coherence tomography," Invest. Ophthalmol. Vis. Sci. 47(12), 54875494 (2006)

24. M. Miura, M. Yamanari, T. Iwasaki, A. E. Elsner, S. Makita, T. Yatagai, and Y. Yasuno, "Imaging polarimetry in age-related macular degeneration," Invest. Ophthalmol. Vis. Sci. 49(6), 2661-2667 (2008).

25. E. Götzinger, M. Pircher, B. Baumann, C. Ahlers, W. Geitzenauer, U. Schmidt-Erfurth, and C. K. Hitzenberger, "Three-dimensional 
polarization sensitive oct imaging, and interactive display of the human retina," Opt. Express 17(5), 4151-4165 (2009).

26. E. Götzinger, M. Pircher, I. Dejaco-Ruhswurm, S. Kaminski, C. Skorpik, and C. K. Hitzenberger, "Imaging of birefringent properties of keratoconus corneas by polarization-sensitive optical coherence tomography," Invest. Ophthalmol. Vis. Sci. 48(8), 3551-3558 (2007).

27. B. Cense, T. C. Chen, B. H. Park, M. C. Pierce, and J. F. de Boer, "Thickness and birefringence of healthy retinal nerve fiber layer tissue measured with polarization-sensitive optical coherence tomography," Invest. Ophthalmol. Vis. Sci. 45(8), 2606-2612 (2004).

28. B. Cense, M. Mujat, T. C. Chen, B. H. Park, and J. F. de Boer, "Polarization-sensitive spectral-domain optical coherence tomography using a single line scan camera," Opt. Express 15, 2421- 2431 (2007).

29. M. Yamanari, M. Miura, S. Makita, T. Yatagai, and Y. Yasuno, "Phase retardation measurement of retinal nerve fiber layer by polarizationsensitive spectral-domain optical coherence tomography and scanning laser polarimetry," J. Biomed. Opt. 13(1), 014013 (2008).

30. E. Götzinger, M. Pircher, B. Baumann, C. Hirn, C. Vass, and C. K. Hitzenberger, "Analysis of the origin of atypical scanning laser polarimetry patterns by polarization-sensitive optical coherence tomography," Invest. Ophthalmol. Vis. Sci. 49(12), 5366-5372 (2008).

31. Y. Yasuno, M. Yamanari, K. Kawana, T. Oshika, and M. Miura, "Investigation of post-glaucoma-surgery structures by three-dimensional and polarization sensitive anterior eye segment optical coherence tomography," Opt. Express 17(5), 3980-3996 (2009).

32. "Opthalmics—corneal topography systems—-standard terminologyrequirements," ANSI Z80.23-1999, American National Standards Institute (1999).

33. L. Kagemann, G. Wollstein, H. Ishikawa, R. A. Bilonick, P. M. Brennen, L. S. Folio, M. L. Gabriele, and J. S. Schuman, "Identification and assessment of Schlemm's canal by spectral domain optical coherence tomography," Invest. Ophthalmol. Vis. Sci. 51(8), 4054-4059 (2010).

34. A. Miyazawa, M. Yamanari, S. Makita, M. Miura, K. Kawana, K. Iwaya, H. Goto, and Y. Yasuno, "Tissue discrimination in anterior eyeusing three optical parameters obtainedby polarization sensitive opticalcoherence tomography," Opt. Express 17(20), 17426-17440 (2009). 